\title{
Thermal convection for a Darcy-Brinkman rotating anisotropic porous layer in local thermal non-equilibrium
}

\author{
Florinda Capone ${ }^{1}\left[\right.$ (1) Jacopo A. Gianfrani ${ }^{1}$ (1) \\ Received: 14 July 2021 / Revised: 2 September 2021 / Accepted: 14 September 2021 / \\ Published online: 16 October 2021 \\ (c) The Author(s) 2021
}

\begin{abstract}
The onset of natural convection in a fluid-saturated anisotropic porous layer, which rotates about the vertical axis, under the hypothesis of local thermal non-equilibrium, is analysed. Since the porosity of the medium is assumed to be high, the more suitable Darcy-Brinkman model is adopted. Linear instability analysis of the conduction solution is carried out. Nonlinear stability with respect to $L^{2}$-norm is performed in order to prove the coincidence between the linear instability and the global nonlinear stability thresholds. The effect of both rotation and thermal and mechanical anisotropies on the critical Rayleigh number for the onset of instability is discussed.
\end{abstract}

Keywords Light anisotropy · Rotating layer · LTNE Darcy-Brinkman model · Linear instability $\cdot$ Energy method

Mathematics Subject Classification 76Dxx $\cdot 76 \mathrm{Exx} \cdot 76 \mathrm{Sxx} \cdot 76 \mathrm{Rxx}$

\section{Introduction}

Thermal convection in fluid-saturated porous media is a research topic of great interest because of its several applications in real life problems, in engineering and geological context. Convection in porous media finds its remarkable attention in geothermal energy utilization, thermal insulation technology, cooling of electronic equipment, tube refrigerators, heat exchangers or more in general, problems on removal or storage of heat.

In industrial field, high-porosity materials, such as metal foams, are usually involved since they can be successfully used to manage heat transfer. Moreover, their light

$凶$ Florinda Capone

florinda.capone@unina.it

1 Department of Mathematics and Applications, Università degli Studi di Napoli "Federico II", Napoli, NA, Italy 
weight, high porosity and rigidity, and a large surface area make them able to recycle energy efficiently. When this kind of materials are taken into account, the usual Darcy model is no longer suitable to describe the fluid motion. The more appropriate DarcyBrinkman model needs to be adopted ([1-4]).

Moreover, many porous media are usually man-made so that they exhibit anisotropy in their mechanical and thermal features. This is because permeability and thermal conductivity can be tuned in order to manage the onset of convection ([5-11]).

Furthermore, in many industrial applications, such as centrifugal filtration processes and rotating machinery, rotation plays an important role on the onset of convection, as well. When investigating these kind of situations where the solid matrix rotates, a rotating frame of reference needs to be introduced [12]. It turns out that a term due to Coriolis acceleration appears in the momentum equation. Many papers dealt with convection in rotating porous media, such as [4,13-17].

As far as thermal convection is concerned, many researchers investigated the problem of convection under the strict assumption of local thermal equilibrium. Of course, this hypothesis is no longer satisfying when heat exchange between fluid and solid matrix is allowed. Therefore, the LTNE (Local Thermal Non-Equilibrium) assumption, which involves two different temperatures $T_{f}$ and $T_{s}$, relative to fluid and solid phase, respectively, becomes relevant. Convection in porous media in local thermal non-equilibrium has received a huge attention by many researchers ([5,18-22]) due to the several applications in real life problems, such as in tube refrigerators, in heat exchangers and in flow in porous metal foams.

This paper is intended to investigate the onset of convection in a rotating highporosity medium, whose mechanical and thermal properties are lightly anisotropic, under the local thermal non-equilibrium assumption. In particular, the influence of rotation and anisotropy on the onset of instability is analysed.

The plan of the paper is the following. In Sect. 2 the Darcy-Brinkman model is introduced and the dimensionless system for perturbation fields is obtained. Section 3 is devoted to the proof of the strong form of the principle of exchange of stabilities, according to which only steady convection can occur. In Sect. 4, the critical Rayleigh number for the onset of convection is determined by performing a linear analysis. While, in Sect. 5 the nonlinear stability analysis of the conduction solution is carried out so as to prove the coincidence between the linear instability threshold and the global nonlinear stability threshold, with respect to the $L^{2}-$ norm. Finally, Sect. 6 deals with numerical simulations that highlight the influence of rotation, anisotropy and the Darcy number on the critical Rayleigh number.

\section{Mathematical model}

Let us take into account a horizontal highly porous medium, whose depth is $d$, saturated by an incompressible, homogeneous fluid at rest. The medium is assumed evenly warmed up from below and rotating about the upward vertical axis $z$ with constant angular velocity $\Omega$. As consequence, in addition to the gravitational field, the Coriolis force acts on the medium. Moreover, we assume that the medium is in local thermal non-equilibrium so that the heat exchange between fluid and solid skeleton is allowed. 
We denote by $T_{L}$ the temperature of the lower plane $z=0$ and by $T_{U}$ the temperature of the upper plane $z=d$, while we refer to the fluid temperature with $T_{f}$ and to the solid temperature with $T_{s}$. Then

$$
T_{s}=T_{f}=T_{L} \quad \text { on } z=0, \quad T_{s}=T_{f}=T_{U} \quad \text { on } z=d
$$

with $T_{L}>T_{U}$.

Besides, we assume that the layer is lightly anisotropic, i.e. its features, such as thermal conductivity and permeability, are homogeneous in the horizontal direction. This assumption allows us to write the permeability tensor $\mathcal{K}^{*}$, the thermal conductivity tensors of solid phase and fluid phase, $\mathcal{D}_{s}^{*}$ and $\mathcal{D}_{f}^{*}$, respectively, in the following way

$$
\begin{array}{lc}
\mathcal{K}^{*}=K_{z} \mathcal{K} & \mathcal{K}=\left(\begin{array}{lll}
\xi & 0 & 0 \\
0 & \xi & 0 \\
0 & 0 & 1
\end{array}\right) \quad \xi=\frac{K_{H}}{K_{z}} \\
\mathcal{D}_{s}^{*}=\kappa_{z}^{s} \mathcal{D}_{s} & \mathcal{D}_{s}=\left(\begin{array}{lll}
\zeta & 0 & 0 \\
0 & \zeta & 0 \\
0 & 0 & 1
\end{array}\right) \quad \zeta=\frac{\kappa_{H}^{s}}{\kappa_{z}^{s}} \\
\mathcal{D}_{f}^{*}=\kappa_{z}^{f} \mathcal{D}_{f} & \mathcal{D}_{f}=\left(\begin{array}{lll}
\eta & 0 & 0 \\
0 & \eta & 0 \\
0 & 0 & 1
\end{array}\right)
\end{array}
$$

being the principal axis $(x, y, z)$ of $\mathcal{K}^{*}$ coinciding with the conductivity tensors' ones.

Since the medium porosity is high, a Darcy-Brinkman model is employed. Accounting for the Oberbeck - Boussinesq approximation, starting from [5]-[19], the model is

$$
\left\{\begin{array}{l}
\mathbf{v}=\mu^{-1} \mathcal{K}^{*} \cdot\left[-\nabla p+\rho_{f} g \alpha T_{f} \mathbf{k}-\frac{2 \Omega \rho_{f}}{\varepsilon} \mathbf{k} \times \mathbf{v}+\tilde{\mu} \Delta \mathbf{v}\right] \\
\nabla \cdot \mathbf{v}=0 \\
\varepsilon(\rho c)_{f} T_{, t}^{f}+(\rho c)_{f} \mathbf{v} \cdot \nabla T_{f}=\varepsilon \nabla \cdot\left(\mathcal{D}_{f}^{*} \cdot \nabla T_{f}\right)+h\left(T_{s}-T_{f}\right) \\
(1-\varepsilon)(\rho c)_{s} T_{, t}^{s}=(1-\varepsilon) \nabla \cdot\left(\mathcal{D}_{s}^{*} \cdot \nabla T_{s}\right)-h\left(T_{s}-T_{f}\right)
\end{array}\right.
$$

where $\mathbf{v}, p, T_{f}$ and $T_{s}$ are (seepage) velocity, reduced pressure, fluid phase temperature and solid phase temperature, respectively; $\tilde{\mu}, \mu, \rho_{f}, \rho_{s}, c, g, \alpha, \Omega, \varepsilon, h$ are effective and dynamic viscosity, fluid density, solid density, specific heat, gravity acceleration, thermal expansion coefficient, angular velocity, porosity and interaction coefficient, respectively.

The following boundary conditions are coupled to (3)

$$
\begin{aligned}
& T_{s}=T_{f}=T_{L} \quad \text { on } z=0, \quad T_{s}=T_{f}=T_{U} \quad \text { on } z=d, \\
& \mathbf{v} \cdot \mathbf{n}=0 \quad \text { on } z=0, d
\end{aligned}
$$

being $\mathbf{n}$ the unit outward normal to planes $z=0, d$.

System (3) admits the following conduction solution $m_{0}$ : 


$$
m_{0}=\left\{\mathbf{v}_{b}=\mathbf{0}, \quad \bar{T}_{s}=\bar{T}_{f}=-\beta z+T_{L}, \quad p_{b}=-\rho_{f} g \alpha \beta \frac{z^{2}}{2}+\rho_{f} g \alpha T_{L} z+c o s t\right\}
$$

where $\beta=\frac{T_{L}-T_{U}}{d}(>0)$ is the adverse temperature gradient.

We are interested in studying the stability of the steady solution (5). Let us introduce the following perturbation fields $\{\mathbf{u}, \theta, \phi, \pi\}$ so as to obtain a new solution for (3)

$$
\mathbf{v}=\mathbf{u}+\mathbf{v}_{b} \quad T_{f}=\theta+\bar{T}_{f} \quad T_{s}=\phi+\bar{T}_{s} \quad p=\pi+p_{b} .
$$

Once the dimensionless quantities are introduced

$$
x_{i}=x_{i}^{*} d, \quad t=t^{*} \frac{\varepsilon d}{U}, \quad \pi=\pi^{*} P, \quad u_{i}=u_{i}^{*} U, \quad \theta=\theta^{*} T^{\prime}, \quad \phi=\phi^{*} T^{\prime}
$$

where

$$
P=\frac{U \mu d}{K_{z}}, \quad U=\frac{\varepsilon \kappa_{z}^{f}}{(\rho c)_{f} d}, \quad T^{\prime}=\beta d \sqrt{\frac{\kappa_{z}^{f} \varepsilon \mu}{\beta g \alpha K_{z} \rho_{f}^{2} c_{f} d^{2}}},
$$

the dimensionless system for the perturbation fields, omitting all the asterisks, is

$$
\left\{\begin{array}{l}
\mathcal{K}^{-1} \cdot \mathbf{u}=-\nabla \pi+R \theta \mathbf{k}-\mathcal{T} \mathbf{k} \times \mathbf{u}+D a \Delta \mathbf{u} \\
\nabla \cdot \mathbf{u}=0 \\
\theta_{, t}+\mathbf{u} \cdot \nabla \theta=R w+\eta \Delta_{1} \theta+\theta_{, z z}+H(\phi-\theta) \\
A \phi_{, t}-\zeta \Delta_{1} \phi-\phi_{, z z}+H \gamma(\phi-\theta)=0
\end{array}\right.
$$

where

$$
\begin{aligned}
\gamma & =\frac{\varepsilon \kappa_{z}^{f}}{(1-\varepsilon) \kappa_{z}^{s}}, \quad A=\frac{(\rho c)_{s} \kappa_{z}^{f}}{(\rho c)_{f} \kappa_{z}^{s}}, \quad H=\frac{h d^{2}}{\varepsilon \kappa_{z}^{f}} \\
R^{2} & =\frac{K_{z} \rho_{f}^{2} c_{f} d^{2} \beta g \alpha}{\mu \varepsilon \kappa_{z}^{f}} \quad \text { Rayleigh number } \\
D a & =\frac{K_{z} \tilde{\mu}}{\mu d^{2}} \text { Darcy number, } \quad \mathcal{T}=\frac{2 \Omega \rho_{f} K_{z}}{\varepsilon \mu} \quad \text { Taylor number. }
\end{aligned}
$$

To system (9) we append the following initial conditions

$$
\mathbf{u}(\mathbf{x}, 0)=\mathbf{u}_{0}(\mathbf{x}), \quad \theta(\mathbf{x}, 0)=\theta_{0}(\mathbf{x}), \quad \phi(\mathbf{x}, 0)=\phi_{0}(\mathbf{x}), \quad \pi(\mathbf{x}, 0)=\pi_{0}(\mathbf{x})
$$

where $\nabla \cdot \mathbf{u}_{0}=0$, and the following stress-free boundary conditions

$$
u_{, z}=v_{, z}=w=\theta=\phi=0 \quad \text { on } z=0,1 .
$$


In order to study the stability of the null solution of (9), let us assume that perturbations are periodic in $x$ and $y$ directions with periods $\frac{2 \pi}{a_{x}}$ and $\frac{2 \pi}{a_{y}}$, respectively. Let

$$
V=\left[0, \frac{2 \pi}{a_{x}}\right] \times\left[0, \frac{2 \pi}{a_{y}}\right] \times[0,1]
$$

be the periodicity cell, we assume that perturbations belong to $W^{2,2}(V), \forall t \in \mathbb{R}^{+}$ and they can be expanded as a Fourier series uniformly convergent in $V$.

\section{Principle of exchange of stabilities}

In order to perform a linear instability analysis, we rewrite $(9)_{1}$ in a more convenient form in which only relevant unknown fields appear. Hence, let us apply the double curl and the curl to (9) 1 and let us retain only the third component. The resulting equations are multiplied by $\xi$ so as to obtain

$$
\left\{\begin{array}{l}
\xi \Delta_{1} w+w_{, z z}=\xi R \Delta_{1} \theta+\xi \mathcal{T}\left(u, y z-v_{, x z}\right)+\xi D a \Delta \Delta w \\
(1-\xi D a \Delta)\left(u, y z-v_{, x z}\right)=-\xi \mathcal{T} w_{, z z}
\end{array}\right.
$$

where $(13)_{2}$ is consequence of a further derivation with respect to $z$. A single equation is obtained once the operator $(1-\xi D a \Delta)$ is applied to $(13)_{1}$ and $(13)_{2}$ is substituted in the resulting equation. This procedure allows us to write the linear version of (9) as follows

$$
\left\{\begin{array}{l}
\xi \Delta_{1} w+w_{, z z}-\xi^{2} D a \Delta \Delta_{1} w-\xi D a \Delta w_{, z z}=\xi R \Delta_{1} \theta \\
\quad-\xi^{2} R D a \Delta \Delta_{1} \theta-\xi^{2} \mathcal{T}^{2} w_{, z z}+\xi D a \Delta \Delta w-\xi^{2} D a^{2} \Delta \Delta \Delta w \\
\theta_{, t}=R w+\eta \Delta_{1} \theta+\theta_{, z z}+H(\phi-\theta) \\
A \phi_{, t}-\zeta \Delta_{1} \phi-\phi_{, z z}+H \gamma(\phi-\theta)=0
\end{array}\right.
$$

Since (14) is autonomous, we look for solutions whose time dependence is separated from the temporal one, i.e.

$$
\hat{\varphi}(t, \mathbf{x})=\varphi(\mathbf{x}) e^{\sigma t} \quad \forall \hat{\varphi} \in(w, \theta, \phi) \quad \sigma \in \mathbb{C}
$$

By virtue of (15), (14) becomes

$$
\left\{\begin{array}{l}
\xi \Delta_{1} w+w_{, z z}-\xi^{2} D a \Delta \Delta_{1} w-\xi D a \Delta w_{, z z}+\xi^{2} R D a \Delta \Delta_{1} \theta \\
\quad-\xi R \Delta_{1} \theta+\xi^{2} \mathcal{T}^{2} w_{, z z}-\xi D a \Delta \Delta w+\xi^{2} D a^{2} \Delta \Delta \Delta w=0 \\
\sigma \theta-R w-\eta \Delta_{1} \theta-\theta_{, z z}-H \phi+H \theta=0 \\
A \sigma \phi-\zeta \Delta_{1} \phi-\phi_{, z z}+H \gamma \phi-H \gamma \theta=0
\end{array}\right.
$$

Then we denote by $(\cdot, \cdot)$ and $\|\cdot\|$ the scalar product on the Hilbert space $L^{2}(V)$, and the related norm, respectively. Let us multiply $(16)_{1}$ by $\frac{w^{*}}{\xi},(16)_{2}$ by 
$(1-\xi D a \Delta) \Delta_{1} \theta^{*}$ and $(16)_{3}$ by $(1-\xi D a \Delta) \Delta_{1} \frac{\phi^{*}}{\gamma}$, where the asterisks denote the complex conjugate. By virtue of boundary conditions (11), integrating over the periodicity cell $V$ yields

$$
\left\{\begin{array}{l}
-\left\|\nabla_{1} w\right\|^{2}-\xi^{-1}\|w, z\|^{2}-\xi D a\left\|\nabla \nabla_{1} w\right\|^{2}-D a\left\|\nabla w_{, z}\right\|^{2} \\
-R\left(\Delta_{1} \theta, w^{*}\right)+\xi R D a\left(\Delta \Delta_{1} \theta, w^{*}\right)-\xi \mathcal{T}^{2}\left\|w_{, z}\right\|^{2} \\
-D a\|\nabla \nabla w\|^{2}-\xi D a^{2}\|\nabla \nabla \nabla w\|^{2}=0
\end{array}\right.
$$

Since $\left(\phi, \Delta_{1} \theta^{*}\right)=\left(\Delta_{1} \phi, \theta^{*}\right)$ and $\left(\phi, \Delta_{1} \theta^{*}\right)=\left(\Delta_{1} \phi, \theta^{*}\right),(17)$ yields

$$
\begin{aligned}
\sigma\left[\left\|\nabla_{1} \theta\right\|^{2}+\xi D a\left\|\nabla \nabla_{1} \theta\right\|^{2}+\frac{A}{\gamma}\left\|\nabla_{1} \phi\right\|^{2}+\frac{A}{\gamma} \xi D a\left\|\nabla \nabla_{1} \phi\right\|^{2}\right]= \\
=-\left\|\nabla_{1} w\right\|^{2}-\xi^{-1}\left\|w_{, z}\right\|^{2}-\xi D a\left\|\nabla \nabla_{1} w\right\|^{2}-D a\left\|\nabla w_{, z}\right\|^{2} \\
\quad-R\left[\left(\Delta_{1} \theta, w^{*}\right)+\left(w, \Delta_{1} \theta^{*}\right)\right]+\xi R D a\left[\left(\Delta \Delta_{1} \theta, w^{*}\right)+\left(w, \Delta_{1} \theta^{*}\right)\right] \\
\quad-\xi \mathcal{T}^{2}\left\|w_{, z}\right\|^{2}-D a\|\nabla \nabla w\|^{2}-\xi D a^{2}\|\nabla \nabla \nabla w\|^{2}-\eta\left\|\Delta_{1} \theta\right\|^{2} \\
-\xi \eta D a\left\|\nabla \Delta_{1} \theta\right\|^{2}-\left\|\nabla_{1} \theta_{, z}\right\|^{2}-\xi D a\left\|\nabla \nabla_{1} \theta_{, z}\right\|^{2} \\
-H\left[\left(\Delta_{1} \phi, \theta^{*}\right)+\left(\theta, \Delta_{1} \phi^{*}\right)\right]+H \xi D a\left[\left(\Delta \Delta_{1} \phi, \theta^{*}\right)+\left(\theta, \Delta \Delta_{1} \phi^{*}\right)\right] \\
-H\left\|\nabla_{1} \theta\right\|^{2}-\xi H D a\left\|\nabla \nabla_{1} \theta\right\|^{2}-\frac{\zeta}{\gamma}\left\|\Delta_{1} \phi\right\|^{2}-\frac{\xi D a \zeta}{\gamma}\left\|\nabla \Delta_{1} \phi\right\|^{2} \\
\quad-\frac{1}{\gamma}\left\|\nabla_{1} \phi_{, z}\right\|^{2}-\frac{\xi D a}{\gamma}\left\|\nabla \nabla_{1} \phi, z\right\|^{2}-H\left\|\nabla_{1} \phi\right\|^{2}-H \xi D a\left\|\nabla \nabla_{1} \phi\right\|^{2}
\end{aligned}
$$

Every term in (18) is real, then necessarily $\sigma$ is real as well. Thus, we have shown the validity of the strong form of the principle of exchange of stabilities. Therefore, convection can occur only through a steady motion. 


\section{Linear instability analysis}

Since our aim is to determine the critical Rayleigh number beyond which instability occurs, we focus on the marginal state in (16). By virtue of principle of exchange of stabilities, we set $\sigma=0$ in (16) so as to obtain

$$
\left\{\begin{array}{c}
\xi \Delta_{1} w+w_{, z z}-\xi^{2} D a \Delta \Delta_{1} w-\xi D a \Delta w_{, z z}-\xi R \Delta_{1} \theta+\xi^{2} R D a \Delta \Delta_{1} \theta \\
\quad+\xi^{2} \mathcal{T}^{2} w_{, z z}-\xi D a \Delta \Delta w+\xi^{2} D a^{2} \Delta \Delta \Delta w=0 \\
-R w-\eta \Delta_{1} \theta-\theta_{, z z}-H \phi+H \theta=0 \\
-\zeta \Delta_{1} \phi-\phi_{, z z}+H \gamma \phi-H \gamma \theta=0
\end{array}\right.
$$

Because of periodicity of perturbation fields, accounting for boundary conditions (11) and since the sequence $\{\sin n \pi z\}_{n \in \mathbb{N}}$ is a complete orthogonal system for $L^{2}([0,1])$, we look for solution of (19) such that

$$
f(x, y, z)=\sum_{n=1}^{+\infty} \bar{f}_{n}(x, y, z) \quad \forall f \in\{w, \theta, \phi\}
$$

where $\bar{f}_{n}=\tilde{f}_{n}(x, y) \sin (n \pi z)$ and

$$
\Delta_{1} \bar{f}_{n}=-a^{2} \bar{f}_{n} \quad \frac{\partial^{2} \bar{f}_{n}}{\partial z^{2}}=-n^{2} \pi^{2} \bar{f}_{n} \quad\left(a^{2}=a_{x}^{2}+a_{y}^{2}\right)
$$

where $a$ is the wavenumber arising from spatial periodicity.

Let us define the following operators

$$
\begin{aligned}
& \mathcal{L}_{1} \equiv \xi \Delta_{1}+\partial_{, z z}-\xi^{2} D a \Delta \Delta_{1}-\xi D a \Delta \partial_{, z z}+\xi^{2} \mathcal{T}^{2} \partial_{, z z}-\xi D a \Delta \Delta \\
&+\xi^{2} D a^{2} \Delta \Delta \Delta \\
& \mathcal{L}_{2} \equiv-\eta \Delta_{1}-\partial_{, z z}+H \\
& \mathcal{L}_{3} \equiv-\zeta \Delta_{1}-\partial_{, z z}+H \gamma
\end{aligned}
$$

so that the system (19) becomes

$$
\left\{\begin{array}{l}
\mathcal{L}_{1} w=(1-\xi D a \Delta) R \xi \Delta_{1} \theta \\
\mathcal{L}_{2} \theta=R w+H \phi \\
\mathcal{L}_{3} \phi=H \gamma \theta
\end{array}\right.
$$

Now let us apply $\mathcal{L}_{1}$ and $\mathcal{L}_{3}$ to $(23)_{2}$ so that

$$
\mathcal{L}_{1} \mathcal{L}_{2} \mathcal{L}_{3} \theta=R \mathcal{L}_{3} \mathcal{L}_{1} w+H \mathcal{L}_{1} \mathcal{L}_{3} \phi
$$

By substituting $(23)_{1}-(23)_{3}$ in (24), we get

$$
\mathcal{L}_{1} \mathcal{L}_{2} \mathcal{L}_{3} \theta=R^{2} \mathcal{L}_{3}(1-\xi D a \Delta) \xi \Delta_{1} \theta+H^{2} \gamma \mathcal{L}_{1} \theta .
$$


By splitting $\mathcal{L}_{3}$ in the first term, we obtain

$$
\begin{array}{r}
\mathcal{L}_{1} \mathcal{L}_{2}\left(-\zeta \Delta_{1}-\partial_{, z z}\right) \theta+\mathcal{L}_{1} H \gamma\left(-\eta \Delta_{1}-\partial_{, z z}\right) \theta \\
=R^{2}\left(-\zeta \Delta_{1}-\partial_{, z z}+H \gamma\right)(1-\xi D a \Delta) \xi \Delta_{1} \theta .
\end{array}
$$

By splitting $\mathcal{L}_{2}$ in the first term in (26)

$$
\begin{gathered}
\mathcal{L}_{1} H\left(-\zeta \Delta_{1}-\partial_{, z z}\right) \theta+\mathcal{L}_{1}\left(-\eta \Delta_{1}-\partial_{, z z}\right)\left(-\zeta \Delta_{1}-\partial_{, z z}+H \gamma\right) \theta \\
=R^{2}\left(-\zeta \Delta_{1}-\partial_{, z z}+H \gamma\right)(1-\xi D a \Delta) \xi \Delta_{1} \theta
\end{gathered}
$$

Let us substitute (20) in (27) and retain only the $n$-th component. Then, from (27)

$$
\begin{aligned}
& {\left[-\xi a^{2}-n^{2} \pi^{2}-\xi^{2} a^{2} D a \delta_{n}-\xi n^{2} \pi^{2} D a \delta_{n}-\xi^{2} \mathcal{T}^{2} n^{2} \pi^{2}-\xi D a \delta_{n}^{2}-\xi^{2} D a^{2} \delta_{n}^{3}\right]} \\
& {\left[H\left(\zeta a^{2}+n^{2} \pi^{2}\right)+\left(\eta a^{2}+n^{2} \pi^{2}\right)\left(\zeta a^{2}+n^{2} \pi^{2}+H \gamma\right)\right]} \\
& =-R^{2}\left(\zeta a^{2}+n^{2} \pi^{2}+H \gamma\right)\left(1+\xi D a \delta_{n}\right) \xi a^{2}
\end{aligned}
$$

being $\delta_{n}=\left(a^{2}+n^{2} \pi^{2}\right)$.

From (28) it follows that the critical Rayleigh number for the onset of steady convection is

$$
R_{S}=\min _{\left(n^{2}, a^{2}\right) \in \mathbb{N} \times \mathbb{R}^{+}} f\left(n^{2}, a^{2}\right)
$$

being

$$
\begin{aligned}
f\left(n^{2}, a^{2}\right)= & \frac{\xi a^{2}+n^{2} \pi^{2}+\xi^{2} D a a^{2} \delta_{n}+\xi D a n^{2} \pi^{2} \delta_{n}+\xi^{2} \mathcal{T}^{2} n^{2} \pi^{2}+\xi D a \delta_{n}^{2}+\xi^{2} D a^{2} \delta_{n}^{3}}{\left(1+\xi D a \delta_{n}\right) \xi a^{2}} \\
& {\left[\eta a^{2}+n^{2} \pi^{2}+H \frac{\zeta a^{2}+n^{2} \pi^{2}}{\zeta a^{2}+n^{2} \pi^{2}+H \gamma}\right] }
\end{aligned}
$$

We can easily remark that the minimum with respect to $n^{2}$ is attained in $n^{2}=1$, since $f\left(\cdot, a^{2}\right)$ is a strictly increasing function. Hence

$$
\begin{aligned}
R_{S}=\min _{a^{2} \in \mathbb{R}^{+}} & {\left[\frac{\xi a^{2}+\pi^{2}+\xi D a \delta^{2}}{\xi a^{2}}+\frac{\xi^{2} \mathcal{T}^{2} \pi^{2}}{(1+\xi D a \delta) \xi a^{2}}\right] . } \\
& {\left[\eta a^{2}+\pi^{2}+H \frac{\zeta a^{2}+\pi^{2}}{\zeta a^{2}+\pi^{2}+H \gamma}\right] }
\end{aligned}
$$

where $\delta=\left(a^{2}+\pi^{2}\right)$.

Let us remark that $f\left(1, a^{2}\right)$ is a strictly increasing function of $\mathcal{T}$ and $\eta$, therefore the stabilizing effect of rotation and fluid thermal conductivity has been proved. In particular, the effect of rotation on the onset of instability is expected since the Coriolis force acts in the horizontal direction, discouraging the motion in the vertical one. 
Moreover, it is easy to note that if $D a=0,(31)$ coincides with the result found in [5]-[19]. While, for a low porous medium in absence of rotation, i.e. $\mathcal{T}=0$ and $D a=0$, results coincide with the ones found in [6]. In addition, by assuming that the layer is isotropic, i.e. $\xi=\eta=\zeta=1$, the critical Rayleigh number (31) is the same as that one found in [18].

\section{Nonlinear stability}

In this section we want to perform a nonlinear stability analysis of conduction solution $m_{0}$. The application of the energy method yields a loss of the rotation term. Therefore, we employ a differential constraint approach $([19,20])$ in order to capture the influence of rotation. Then, let us multiply $(9)_{3}$ by $\theta$ and $(9)_{4}$ by $\phi$ and integrate over the periodicity cell $V$. By defining the Lyapunov functional $E(t)$

$$
E(t)=\frac{1}{2}\|\theta\|^{2}+\frac{A}{2 \gamma}\|\phi\|^{2},
$$

the production term $I(t)$

$$
I(t)=(\theta, w)
$$

and the dissipation function $D(t)$

$$
D(t)=\eta\left\|\nabla_{1} \theta\right\|^{2}+\left\|\theta_{, z}\right\|^{2}+\frac{\zeta}{\gamma}\left\|\nabla_{1} \phi\right\|^{2}+\frac{1}{\gamma}\left\|\phi_{, z}\right\|^{2}+H\|\phi-\theta\|^{2}
$$

we find out that

$$
\frac{d E}{d t}=-D\left(1-R \frac{I}{D}\right) \leq-D\left(1-\frac{R}{R_{E}}\right)
$$

where

$$
\frac{1}{R_{E}}=\max _{\mathcal{H}^{*}} \frac{I}{D}
$$

with

$$
\begin{aligned}
& \mathcal{H}^{*}=\{(w, \theta, \phi): w=\theta=\phi=0 \text { on } z=0,1 \text {; periodic in } x \text { and } y \\
& \text { directions, with period } \left.\frac{2 \pi}{a_{x}}, \frac{2 \pi}{a_{y}} \text { respectively; } D<\infty ; \text { verifying }(16)_{1}\right\}
\end{aligned}
$$

the space of the kinematically admissible perturbations. Equation (35) tells us that $R<R_{E}$ is a sufficient condition for the nonlinear stability of $m_{0}$. 
Let us define

$$
\begin{aligned}
g(\mathbf{x})= & \xi \Delta_{1} w+w_{, z z}-\xi^{2} D a \Delta \Delta_{1} w-\xi D a \Delta w_{, z z}-\xi R \Delta_{1} \theta \\
& +\xi^{2} R D a \Delta \Delta_{1} \theta+\xi^{2} \mathcal{T}^{2} w_{, z z}-\xi D a \Delta \Delta w+\xi^{2} D a^{2} \Delta \Delta \Delta w \\
\mathcal{H}= & \{(w, \theta, \phi): w=\theta=\phi=0 \text { on } z=0,1 ; \text { periodic in } x \text { and } y \\
& \text { directions, with period } \left.\frac{2 \pi}{a_{x}}, \frac{2 \pi}{a_{y}} \text { respectively; } D<\infty\right\} .
\end{aligned}
$$

The variational problem (36) is equivalent to

$$
\frac{1}{R_{E}}=\max _{\mathcal{H}} \frac{I+\int_{V} \lambda g d V}{D}
$$

where $\lambda(\mathbf{x})$ is a Lagrange multiplier.

By employing the Poincaré inequality in (34), we get

$$
D(t) \geq a \pi^{2}\|\theta\|^{2}+b \frac{\pi^{2}}{\gamma}\|\phi\|^{2}
$$

being $a=\min \{1, \eta\}$ and $b=\min \{1, \zeta\}$. Hence, if $R<R_{E}$, from (35) it turns out that

$$
\frac{d E}{d t} \leq-\left(a \pi^{2}\|\theta\|^{2}+b \frac{\pi^{2}}{\gamma}\|\phi\|^{2}\right)\left(1-\frac{R}{R_{E}}\right) \leq-\pi^{2}\left(\frac{R_{E}-R}{R_{E}}\right) c E(t)
$$

where $c=\min \left\{2 a, \frac{2 b}{A}\right\}$. Equation (41) yields the exponential decay of temperature perturbation fields.

Now let us remark that, by multiplying (9) 1 by $\mathbf{u}$ and by virtue of Cauchy-Schwartz, we obtain the exponential decay of $\mathbf{u}$, i.e.

$$
\|\mathbf{u}\|^{2} \leq \xi_{*} R^{2}\|\theta\|^{2}
$$

where $\xi_{*}=\max \{\xi, 1\}$.

Thus, we have shown that the condition $R<R_{E}$ implies the global nonlinear and exponential stability of conduction solution $m_{0}$. 
Now let us solve the variational problem (39) to determine the critical Rayleigh number $R_{E}$. The Euler-Lagrange equations, together with the constraint equation, are

$$
\left\{\begin{aligned}
\xi \Delta_{1} w+ & w_{, z z}-\xi^{2} D a \Delta \Delta_{1} w-\xi D a \Delta w_{, z z}-\xi R_{E} \Delta_{1} \theta+\xi^{2} \mathcal{T}^{2} w_{, z z} \\
& +\xi^{2} R_{E} D a \Delta \Delta_{1} \theta-\xi D a \Delta \Delta w+\xi^{2} D a^{2} \Delta \Delta \Delta w=0 \\
\theta+\xi \Delta_{1} \lambda+\lambda, z z-\xi^{2} D a \Delta \Delta_{1} \lambda-\xi D a \Delta \lambda_{, z z}+\xi^{2} \mathcal{T}^{2} \lambda, z z-\xi D a \Delta \Delta \lambda & \\
& +\xi^{2} D a^{2} \Delta \Delta \Delta \lambda=0 \\
R_{E} w+ & \xi^{2} R_{E}^{2} D a \Delta \Delta_{1} \lambda-\xi R_{E}^{2} \Delta_{1} \lambda+2 \eta \Delta_{1} \theta+2 \theta_{, z z}+2 H(\phi-\theta)=0 \\
\zeta \Delta_{1} \phi & +\phi_{, z z}-H \gamma(\phi-\theta)=0
\end{aligned}\right.
$$

Recalling the definitions in (22), (43) becomes

$$
\left\{\begin{array}{l}
\mathcal{L}_{1} w=(1-\xi D a \Delta) R_{E} \xi \Delta_{1} \theta \\
\mathcal{L}_{1} \lambda=-\theta \\
2 \mathcal{L}_{2} \theta=R_{E} w+2 H \phi-(1-\xi D a \Delta) R_{E}^{2} \xi \Delta_{1} \lambda \\
\mathcal{L}_{3} \phi=H \gamma \theta
\end{array}\right.
$$

By applying $\mathcal{L}_{1}$ to $(44)_{3}$ and substituting $(44)_{2}$ and (44) 1 , we obtain

$$
-\mathcal{L}_{1} \mathcal{L}_{2} \theta+H \mathcal{L}_{1} \phi+R_{E} \mathcal{L}_{1} w=0
$$

The application of $\mathcal{L}_{3}$ to (45) leads to the following equation

$$
-\mathcal{L}_{1} \mathcal{L}_{2} \mathcal{L}_{3} \theta+H \mathcal{L}_{1} \mathcal{L}_{3} \phi+R_{E} \mathcal{L}_{3} \mathcal{L}_{1} w=0
$$

which coincides with (24). As consequence, we have obtained the coincidence between the global nonlinear stability threshold $R_{E}$ and the linear instability threshold $R_{S}$, implying the absence of subcritical instability region. This result allows us to claim that the condition $R<R_{E}=R_{S}$ is a necessary and sufficient condition for the stability of $m_{0}$, therefore in this respect the result is optimal.

\section{Numerical analysis}

In this section we would like to point out how parameters affect the onset of convection. Given the complex expression obtained in (31) for the critical Rayleigh number $R_{S}$, it is not always easy to show analytically how parameters modify the occurrence of instability. That is why the expression for the critical Rayleigh number (31) is analysed numerically for different values of parameters with the aim of highlighting how they affect the onset of convection. In particular, we will show the dependence of $R_{S}$ with respect to the Taylor number, permeability, thermal conductivities and the Darcy number.

We would like to point out that results are reported as function of the inter-phase heat transfer coefficient $H$. This parameter is not easily measurable, as claimed in [5]. 


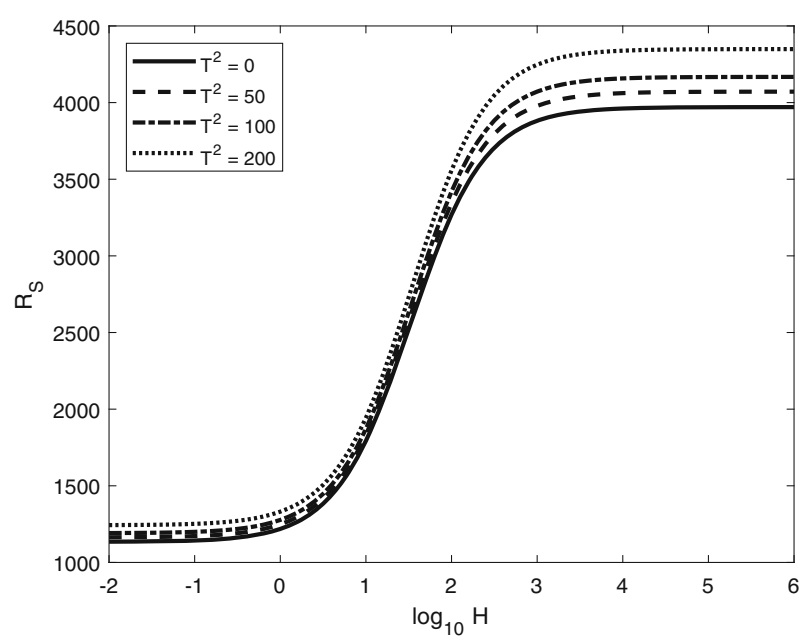

Fig. 1 Critical Rayleigh number as function of the inter-phase heat transfer coefficient $H$ for different values of the Taylor number $\mathcal{T}$ with $\xi=\zeta=\eta=0.5, \gamma=0.4$ and $D a=2$

That is why we have decided to fix the range $\left(10^{-2}, 10^{6}\right)$ in which $H$ can vary and to show the influence of parameters on $R_{S}$ for any $H$.

Figure 1 shows the behaviour of the critical Rayleigh number $R_{S}$ for increasing values of $\mathcal{T}$. The stabilizing effect of rotation on the onset of convection is clear, for any value of $H$, even though it is less remarkable when $H \rightarrow 0$. This result is not surprising since we have previously pointed out that the derivative of $R_{S}$ in (31) with respect to $\mathcal{T}$ is strictly positive. Moreover, the stabilizing effect is expected from a physical point of view since rotation acts on the fluid in the horizontal direction, making the motion along the vertical axis more difficult.

We would like to underline that both for small and large values of $H$, the curves tend to become parallel to the $x$ axis. The region where a plateau is reached represents the local thermal equilibrium situation and it will characterize the following figures, as well. Physically, if $H \rightarrow 0$, the solid phase is separated from the fluid one and it ceases to affect the fluid thermal field. While, if $H \rightarrow \infty$, solid and fluid temperature end up with being identical.

In Fig. 2, the stabilizing effect of the Darcy number $D a$ on conduction is highlighted. Note that if $D a=0$, results are valid for the classical Darcy model, as pointed out previously. It is well known that when porosity $\varepsilon$ tends to 1 , the classical Darcy model needs to be replaced by the Darcy-Brinkman model, for which $D a \neq 0$. Since the Darcy-Brinkman model is closer to a model describing the fluid motion in absence of porous medium (clear fluid), for which it is common knowledge that the critical Rayleigh number is greater than that one in a porous medium, result in Fig. 2 is consistent.

In Fig. 3a, the behaviour of $R_{S}$ with respect to permeability parameter $\xi$ is shown. The destabilizing effect of permeability is evident, for any values of $H$, in agreement with findings of [5] and [6]. Recalling the definition of the Rayleigh number, this kind of behaviour is expected, since $R$ is directly proportional to $K_{z}$. Physically, increasing 


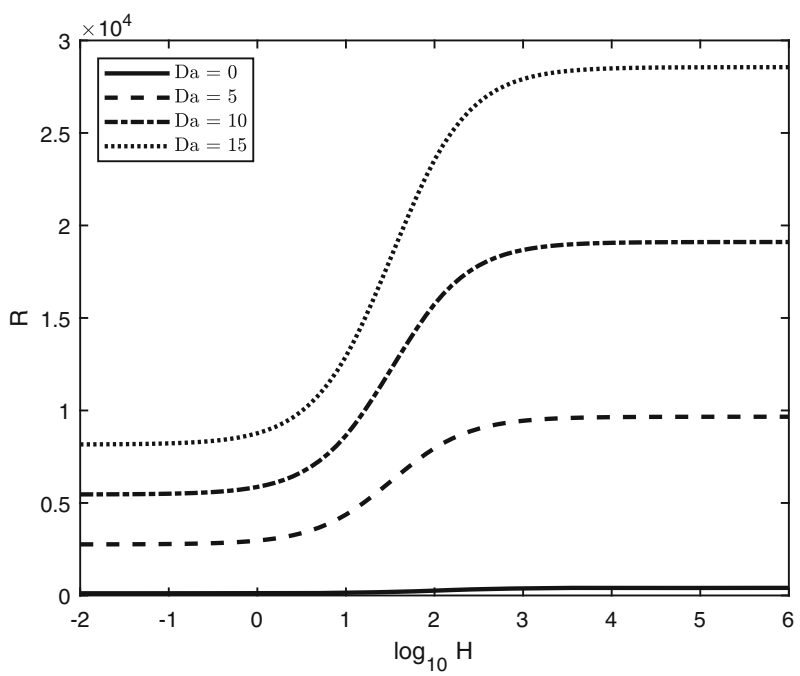

Fig. 2 Critical Rayleigh number as function of the inter-phase heat transfer coefficient $H$ for different values of the Darcy number $D a$ with $\xi=\zeta=\eta=0.5, \gamma=0.4$ and $\mathcal{T}^{2}=20$

(a)

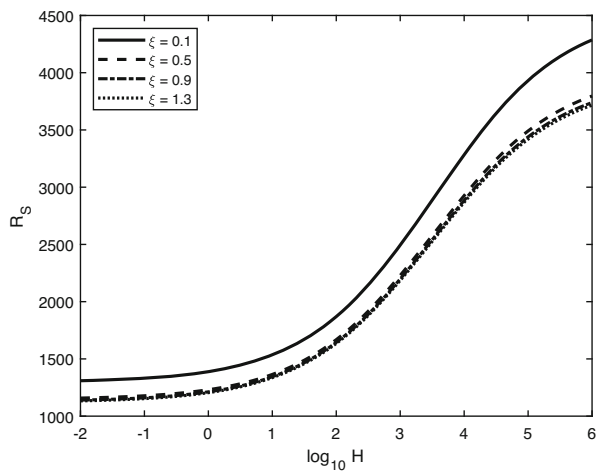

(b)

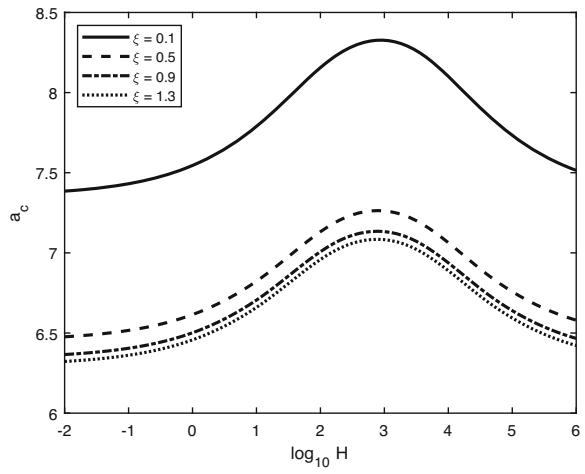

Fig. 3 a Critical Rayleigh number $\mathbf{b}$ critical wavenumber as function of the inter-phase heat transfer coefficient $H$ for different values of $\xi$ with $\zeta=\eta=0.5, \gamma=0.4, \mathcal{T}^{2}=20$ and $D a=2$

$K_{H}$ eases the fluid motion in the horizontal direction, implying an easier occurrence of instability. Figure $3 \mathrm{~b}$ shows how cell dimension changes for different values of permeability. In particular, when permeability in the horizontal direction $K_{H}$ grows, periodicity cells get wider.

Now let us analyse how $R_{S}$ varies with respect to thermal conductivities. Figure $4 \mathrm{a}$ shows the stabilizing effect of solid thermal conductivity on conduction. The growth of $R_{S}$ with $\zeta$ means that solid thermal conductivity delays the onset of convection. Physically, the greater the solid thermal conductivity is the more easily the solid matrix absorbs heat from the fluid. The delaying effect is evident for large values of the interphase heat transfer coefficient $H$, while it is less remarkable for smaller values of $H$. 
(a)

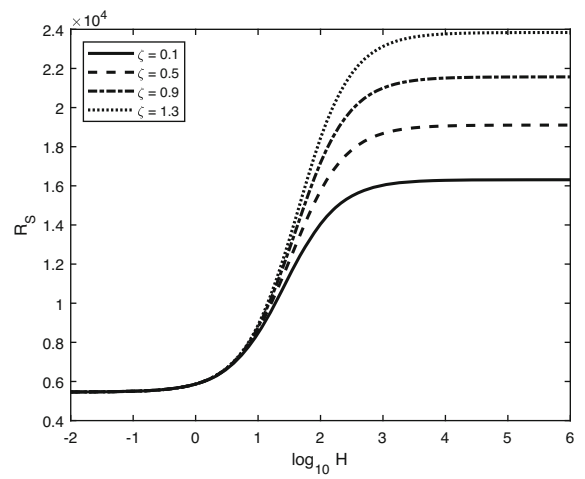

(b)

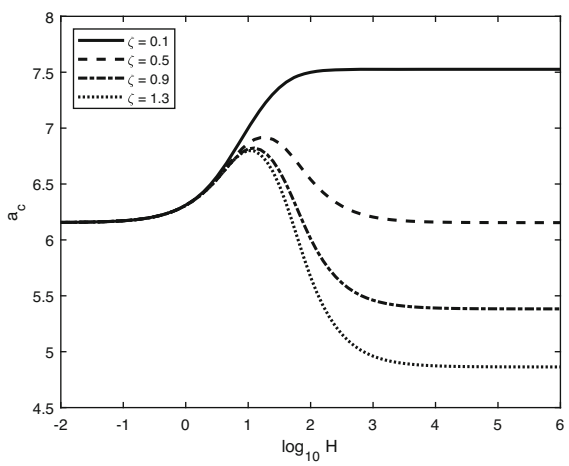

Fig. 4 a Critical Rayleigh number $\mathbf{b}$ critical wavenumber as function of the inter-phase heat transfer coefficient $H$ for different values of $\zeta$ with $\xi=\eta=0.5, \gamma=0.4, \mathcal{T}^{2}=20$ and $D a=10$

(a)

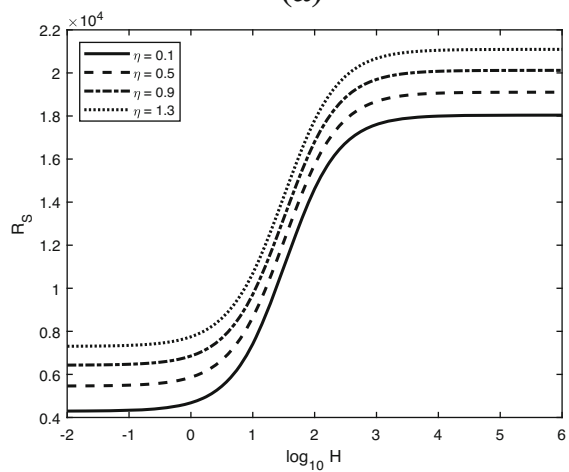

(b)

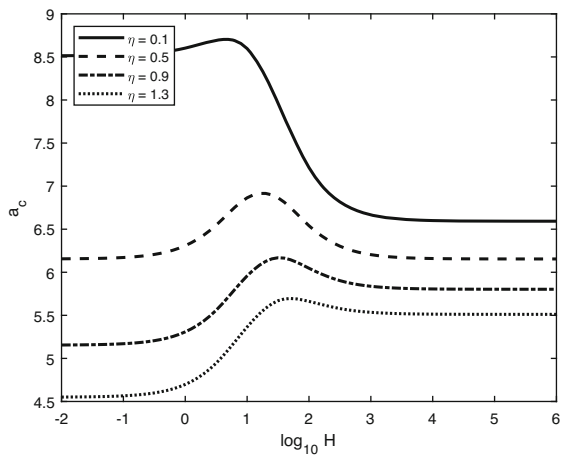

Fig. 5 a Critical Rayleigh number $\mathbf{b}$ critical wavenumber as function of the inter-phase heat transfer coefficient $H$ for different values of $\eta$ with $\xi=\zeta=0.5, \gamma=0.4, \mathcal{T}^{2}=20$ and $D a=10$

This is not surprising since, as already pointed out, when $H \rightarrow 0$ solid phase does not affect the fluid thermal field. This behaviour is evident also in Figure $4 b$, where it is highlighted how cell dimension varies with respect to $\zeta$. In particular, for small values of $H$, the influence is negligible, while if $H$ is great, $\zeta$ promotes wider periodicity cells.

Analogous result is obtained when looking at the effect of the fluid thermal conductivity parameter $\eta$ on the periodicity cell dimension. Figure $5 \mathrm{~b}$ shows that $\eta$ promotes wider periodicity cells, as well.

The stabilizing effect of $\eta$ is highlighted in Fig. 5a, where for any $H, R_{S}$ grows for increasing $\eta$. This behaviour is consistent with the analytical result for which the derivative of $R_{S}$ in (31) with respect to $\eta$ is strictly positive. From a physical point of view, increasing $\kappa_{z}^{f}$ implies that heat flows easier in the vertical direction within the fluid, fostering the onset of instability. 


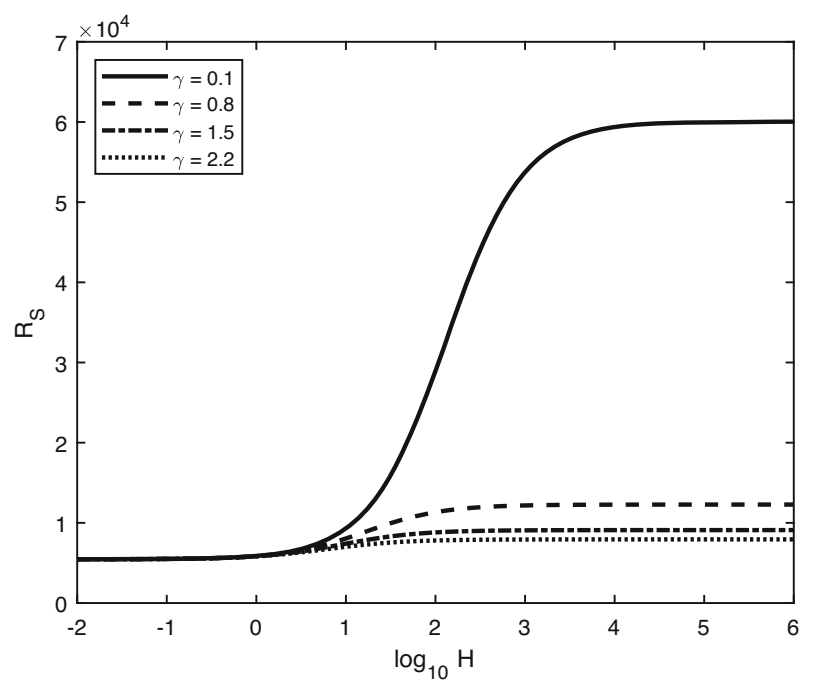

Fig. 6 Critical Rayleigh number as function of the inter-phase heat transfer coefficient $H$ for different values of $\gamma$ with $\xi=\zeta=\eta=0.5, \mathcal{T}^{2}=20$ and $D a=10$

The effect of $\kappa_{z}^{f}$ on the critical Rayleigh number is evident also by looking at Fig. 6 , where the behaviour of $R_{S}$ with respect to the thermal conductivities' ratio $\gamma$ is shown. Since $\gamma=\frac{\varepsilon \kappa_{z}^{f}}{(1-\varepsilon) \kappa_{z}^{s}}$ by definition, increasing $\kappa_{z}^{f}$ implies growing $\gamma$, which yields a decrease for $R_{S}$, i.e. a destabilizing effect, as shown in Fig. 6.

\section{Conclusions}

The linear and nonlinear stability analysis of the conduction solution in an anisotropic rotating porous medium with high porosity in local thermal non-equilibrium has been studied. Only steady convection is allowed, since the principle of exchanges of stability holds. A detailed proof of that has been performed. In order to study the global nonlinear stability, the energy method has been adopted. Coincidence between the linear instability and the global nonlinear stability thresholds is proved. This means that a necessary and sufficient condition for the onset of convection has been obtained.

Numerical simulations have been required in order to highlight the influence of parameters on the onset of convection. It has been pointed out that permeability has a destabilizing effect on conduction. This is because increasing permeability eases the fluid motion, implying an easier occurrence of instability. Whereas, thermal conductivities stabilize conduction, delaying the onset of convection. Moreover, it has been shown that, as expected, rotation and the Darcy number have a stabilizing effect on conduction, as well as the thermal conductivities' ratio $\gamma$. 
Acknowledgements This paper has been performed under the auspices of the National Group of Mathematical Physics GNFM-INdAM. J. Gianfrani would like to thank Progetto Giovani GNFM 2020: "Problemi di convezione in nanofluidi e in mezzi porosi bidispersivi". The Authors would like to thank the anonymous reviewer for the helpful suggestions that led to improvement in the manuscript.

Author Contributions Not applicable.

Funding Open access funding provided by Università degli Studi di Napoli Federico II within the CRUICARE Agreement.

Data Availibility Statement Not applicable.

\section{Declarations}

Conflicts of interest/Competing interests: The authors declare that they have no conflicts of interest.

Code availability: Not applicable.

Open Access This article is licensed under a Creative Commons Attribution 4.0 International License, which permits use, sharing, adaptation, distribution and reproduction in any medium or format, as long as you give appropriate credit to the original author(s) and the source, provide a link to the Creative Commons licence, and indicate if changes were made. The images or other third party material in this article are included in the article's Creative Commons licence, unless indicated otherwise in a credit line to the material. If material is not included in the article's Creative Commons licence and your intended use is not permitted by statutory regulation or exceeds the permitted use, you will need to obtain permission directly from the copyright holder. To view a copy of this licence, visit http://creativecommons.org/licenses/by/4.0/.

\section{References}

1. Capone, F., Rionero, S.: Brinkman viscosity action in porous mhd convection. Int. J. Non-Linear Mech. 85, 109-117 (2016)

2. Capone, F., DeLuca, R.: Onset of convection for ternary fluid mixtures saturating horizontal porous layers with large pores. Atti della Accademia Nazionale dei Lincei, Classe di Scienze Fisiche, Matematiche e Naturali, Rendiconti Lincei Matematica e Applicazioni 23(4), 405-428 (2012)

3. Capone, F., De Luca, R.: Ultimately boundedness and stability of triply diffusive mixtures in rotating porous layers under the action of brinkman law. Int. J. Non-Linear Mech. 47(7), 799-805 (2012)

4. Capone, F., De Luca, R.: On the stability-instability of vertical throughflows in double diffusive mixtures saturating rotating porous layers with large pores. Ricerche di Matematica 63(1), 119-148 (2014)

5. Govender, S., Vadasz, P.: The effect of mechanical and thermal anisotropy on the stability of gravity driven convection in rotating porous media in the presence of thermal non-equilibrium. Transp. Porous Media 69, 55-66 (2007)

6. Malashetty, M.S., Shivakumara, I.S., Kulkarni, S.: The onset of convection in an anisotropic porous layer using a thermal non-equilibrium model. Transp. Porous Media 60, 199-215 (2005)

7. Shivakumara, I.S., Lee, J., Mamatha, A.L., Ravisha, M.: Boundary and thermal non-equilibrium effects on convective instability in an anisotropic porous layer. J. of Mech. Sci. Technol. 25(4), 911-921 (2011)

8. Tyvand, P.A., Storesletten, L.: Onset of convection in an anisotropic porous layer with vertical principal axes. Transp. Porous Media 108(3), 581-593 (2015)

9. Storesletten, L., Rees, D.A.S.: An analytical study of free convective boundary layer flow in porous media:The effect of anisotropic diffusivity. Transp. Porous Media 27, 289-304 (1997)

10. Capone, F., Gentile, M., Hill, A.A.: Penetrative convection via internal heating in anisotropic porous media. Mech. Res. Commun. 37(5), 441-444 (2010)

11. Capone, F., Gentile, M., Hill, A.A.: Convection problems in anisotropic porous media with nonhomogeneous porosity and thermal diffusivity. Acta applicandae mathematicae 122(1), 85-91 (2012)

12. Vadasz, P.: Instability and convection in rotating porous media: a review. Fluids 4, 147 (2019)

13. Vadasz, P.: Fluid flow and heat transfer in rotating porous media. Springer, Newyork (2016) 
14. F. Capone, R. De Luca, M. Gentile Coriolis effect on thermal convection in a rotating bidispersive porous layer Proceedings of the royal society a: mathematical, physical and engineering sciences 476(2235) (2020)

15. F. Capone, R. De Luca, M. Gentile Thermal convection in rotating anisotropic bidispersive porous layers Mech. Res. Commun. 110 (2020)

16. Vadasz, P.: Coriolis effect on gravity-driven convection in a rotating porous layer heated from below. J. of Fluid Mech. 376, 351-375 (1998)

17. P. Vadasz Heat transfer and fluid flow in rotating porous media Developments in Water Science, vol 47, Elsevier, London pp 469-476 (2002)

18. Banu, N., Rees, D.A.S.: Onset of darcy-bénard convection using a thermal non-equilibrium model. Int J Heat Mass Transfer 45, 2221-2228 (2002)

19. Capone, F., Gentile, M.: Sharp stability results in LTNE rotating anisotropic porous layer. Int. J. Therm. Sci. 134, 661-664 (2018)

20. B. Straughan Global nonlinear stability in porous convection with a thermal non-equilibrium model Proc. R. Soc. A 462, 409-418 (2006)

21. Malashetty, M.S., Swamy, M.: Effect of rotation on the onset of thermal convection in a sparsely packed porous layer using a thermal non-equilibrium model. Int. J. Heat and Mass Transf. 53, 30883101 (2010)

22. Celli, M., Barletta, A., Rees, D.A.S.: Local thermal non-equilibrium analysis of the instability in a vertical porous slab with permeable sidewalls. Transport in Porous Media 119(3), 539-553 (2017)

Publisher's Note Springer Nature remains neutral with regard to jurisdictional claims in published maps and institutional affiliations. 